\title{
Relevance of Energy Efficiency Gain in Massive MIMO Wireless Network
}

\author{
Ahmed Alzahrani, Vijey Thayananthan, Muhammad Shuaib Qureshi \\ Computer Science Department, Faculty of Computing and Information Technology \\ King Abdulaziz University, Jeddah 21589, Saudi Arabia.
}

\begin{abstract}
The massive MIMO and energy efficiency (EE) analysis for the next generation technology are the hottest topics in wireless network research. This paper explains about massive MIMO wireless networks and EE for manifold channel which is an evolution massive MIMO. This research will help to design and implement a practical system of next generation network based on massive MIMO where efficient processing provides EE gain. In order to approach this research, different types of manifolds are considered with efficient techniques that depend on the rank of the channel matrix. Employing the specific manifold that helps to analyze the rate of the feedback increases not only the overall performance of the MIMO system but also the EE. We studied the convergence techniques used for optimizing quantization errors which have influences with manifold feedback. Here, we have focused on relevant areas which are very important to analyze $\mathrm{EE}$ gain in the future massive network. According to the selected results obtained in this research, many challenges will be possible to make useful proposals.
\end{abstract}

Keywords-Massive MIMO; manifolds; EE gain; feedback; convergence; quantization

\section{INTRODUCTION}

Concept of basic MIMO system has been used in many applications for many decades but its design concept used in wireless networks is growing with next generation technology. The EE concepts are very attractive because they are not only used for the cost reductions, but also they are very useful to increase the lifetime of the components used in massive MIMO wireless networks.

Massive MIMO wireless networks and communication systems are implemented with a large number of antennas in multi-channel environments [1]. In this, channel used in massive MIMO should be maintained because EE gain depends on the dynamic and statistic behavior of the channel. If the channel state information (CSI) is considered, the feedback employed in the receiver should be optimized to improve EE performance. The optimal feedback design using appropriate manifolds and massive MIMO can be analyzed through energy-efficient algorithms depended on the channel matrix, which needs a manifold to improve the rank and dimension of the covariance matrix [3], [4]. Complexity is a serious concern for massive MIMO because the dimension of the matrix is influenced directly.

The novelty of this research is manifold feedback designed for massive MIMO system. Regarding the EE improvement based on massive MIMO, analyzing the key observations of massive MIMO such as feedback and few relevant parameters are useful in this research.

As a practical matter, however, this EE excitement is tempered by the feasibility of making perfect and global CSI available at all the terminals of a massive MIMO network. It is very difficult and often impossible to provide such a perfect CSI because of rapid channel variations and power increasing dimensionality of channels. In the massive MIMO scheme, the uncertainty of channel estimates even at the receiver, and limited availability of communication resources to send information back from the receiver to other terminals in a network. Thus, it is critical to developing techniques for providing limited CSI with reasonable EE to the transmitters through the feedback used massive MIMO scheme [9]. This limited CSI at the transmitters helps significantly to boost the capabilities of current wireless technologies.

When considering a single user communication and a point-to-point link in the massive MIMO scheme, utilization of antennas is unavoidable. Thus, the transmitter and receiver needs $N_{t}$ and $N_{r}$ antennas respectively. The practical problem of the conventional receiver is always a big issue in the design. Here, manifold techniques provide big improvements when design of the feedback is efficient. Overhead problem and EE is increased with a total number of antennas used in the transmitter of massive MIMO [6]. The geodesic and chordal distances considered in the manifold of dynamic channel should be appropriate to make efficient calculations. The optimized with dimensions of the manifold which MIMO channel needs to reduce the rank of the channel matrix, power and complexity. A geodesic, which is a non-linear curve of MIMO channel, holds a distance between any two points located on the curve assumed as a surface of any manifolds [2]. This small curve imagined on the manifold of the channel is a straight line. Also these two points are very close to each other in the Euclidean space.

Singular value decomposition (SVD) of the matrix and eigenvectors associated to this matrix are necessary steps to calculate the performance of massive MIMO systems. These steps and calculations in MIMO need to be improved through the appropriate optimization techniques [8]. Linear channel model based on SVD with optimization will enhance the EE in an open and closed loop of MIMO system.

The sections and sub-sections in the paper are categorized as follows. Section II provides basic information of MIMO and design that enhances the EE. Section III introduces the massive MIMO with feedback and types of manifolds. In Section IV, 
study of feedback and brief convergence techniques for EE gain are given. Results and analysis of this research based on Pn-manifold and Stiefel manifold are tabulated and discussed with necessary graphs in Section V. Overall conclusions, and further work are summarized in Section VI.

\section{DESIGN OF MIMO}

According to [18], the design of transmitter and receiver are analyzed for MIMO system. The design of the transmitter allows us to increase the $\mathrm{EE}$ and rate with same transmission power. It means that it requires less transmission energy for higher data rate. MIMO system requires more energy than SISO because circuit and signal processing for MIMO require large energy consumption.

\section{A. Basic MIMO}

The basic MIMO contains transmitter and receiver with multiple transmitting and receiving antennas, respectively. In MIMO system model, the parameters of the precoding $P_{i}$, input $X_{i}$, channel $H_{i}$, noise $\eta_{i}$ and output $Y_{i}$ are used to form a mathematical model.

$$
Y_{i}=H_{i} P_{i} X_{i}+\eta_{i}
$$

In this system (1), following dimensions are used, $\quad P_{i} \in$ $\mathbb{F}^{N_{r} \times N_{r}}, X_{i} \in \mathbb{F}^{N_{t}}, \eta_{i} \in \mathbb{F}^{N_{r}}, H_{i} \in \mathbb{F}^{N_{r} \times N_{t}}$ and $Y_{i} \in \mathbb{F}^{N_{r}}$. In order to calculate the $\mathrm{EE}$ gain for fixed and variable rate of MIMO system, statistic power requirements of the internal components and dynamic power of communication channels are required. If total data $n$ is transmitted through MIMO with same transmission power $P_{t}$ and data rate $\mathrm{R}$, the transmission energy can be calculated as

$$
E_{t}=\frac{n P_{t}}{R}
$$

Matrices influenced to the channel are varied with the time, external noise and other environmental changes. So, design of basic MIMO and its channel matrices should be able to handle all situations with low energy consumptions.

Spectral and EE limits of single and multiple links that handle in different environmental conditions can also be designed in massive MIMO [15].

\section{B. Application of MIMO}

Applications of MIMO are many, but they can be categorized into three different approaches they are coordinated MIMO, massive MIMO and millimeter wave MIMO. In network MIMO, coordinated MIMO is used for which EE influences with improvement of spectral efficiency and specific coordination [14], [17]. Massive MIMO is increasing with a number of users and base stations where EE increases with a number of antennas [5]. Millimeter wave MIMO may be considered in the $5 \mathrm{G}$ development. According to the research [10], the manifolds can be used in all energy saving applications involved with non-linear channel models.

\section{Basic MIMO with Manifolds}

The manifolds can be used in the feedback of the basic MIMO. Within the receiver, manifolds are employed to optimize the EE and power which increases the overall performance. Here, energy is analyzed with feedback optimization using Stiefel manifolds. In feedback, all available manifolds are considered to increase the overall performance of basic MIMO [12]. In order to optimize the energy performance, the Stiefel manifold can be used in MIMO, channel estimations and some specific modulations schemes.

\section{Design of Energy Efficient MIMO with Manifolds}

Energy efficient MIMO can be designed from various ways they are such as reducing complexity during the processing and efficient energy managements. The complexity of MIMO depends on the algorithms used in the communication path during the processing. To increase the EE, properties of the manifold such as rank reduction, optimization can be used dynamically.

\section{DESIGN OF MASSIVE MIMO}

Massive MIMO is designed with a large number of antennas which may be either fixed or variable size. Gain of each antenna is also considered with sizes of antennas (gain is proportional to the area of the antenna). When employing the energy efficient components used in the massive MIMO system, the overall lifetime increases. The overall EE gain depends on the design dedicated for particular applications. When more features and services are involved in a particular application, energy consumption will increase but best EE can be achieved with optimum design. It is also of interest to study structured quantization codebooks for feedback that helps us to design massive MIMO with low decoding complexity.

\section{A. Massive MIMO with Feedback}

The design concept of feedback used in the massive MIMO is same as basic MIMO. Here, the size of the channel matrix is very large for the feedback that carries CSI to the base station (BS). Processing of passing CSI through the feedback needs energy which depends on the number of antennas used in BS. When increasing the number of antennas at BS, progress of feedback changes to handle the CSI. Here, energy consumption is proportional to the size reduction which is possible with matrix techniques employed in feedback. Regarding the EE analysis, following types are studied.

1) Perfect or limited CSI is available at the transmitter

2) Perfect or limited feedback channel

3) Linear precoding based on CSI

The energy of the feedback channel is the ratio of the power used in the feedback and the data rate on the feedback channel as given (2). Here, sum of the source coding rate is also in which feedback channel uses the optimization. In this paper, a low-rate, Zero-Delay, error-free feedback channel from the receiver to the transmitter are basic assumptions. It means that the receiver is assumed to have perfect channel knowledge. Here, achieving EE in massive MIMO is a big challenge.

In particular, challenges of feedback employed in massive MIMO are expected to compute from geometric parameters like dimension, coordinates, geodesic distance, manifold volume and ball volumes for various types of manifolds defined through equality or inequality constraints on traces and equality or inequality constraints on ranks of the matrices. 
Using these parameters, we can analyze the EE performance of sphere packing quantization codebooks and random quantization codebooks over those manifolds. Our objective in this project would be to use those parameters to design spectrally efficient MIMO systems with quantized covariance feedback [6], [7] that increases the EE.

\section{B. Types of Manifolds with Feedback}

In the recent research papers, manifolds are considered with latest MIMO schemes that use the energy efficient feedback designed with following manifold techniques.

- Grassmannian: The special structure of the Grassmann manifold that affects the capacity of the overall MIMO system including massive MIMO. Here, EE depends on the rank and dimension of the channel matrix obtained from the massive MIMO and used for manifold technique. Some useful information about Grassmannian manifold and its quantization bounds, which affect the capacity and EE in feedback design, are studied from [10].

- Riemannian: In a complete Riemannian view, distance between the two selected points along the geodesic curve should be optimized. Geodesic curves and optimized distances around the manifold are what channel matrix expects to minimize the dimension and increases the smoothness of the curve used around the manifold. Minimizing dimension helps us to increase the EE in feedback design.

- Pn-manifold: The covariance matrix is computed from the current channel used between the transmitter and receiver antennas [7]. The covariance matrix is represented by a point on the surface of any $3 \mathrm{D}$ objects called as Pn-manifold. The EE of feedback depends on the number of bits used in the codebook, which is a representation of quantization created from Pnmanifolds [7].

- Stiefel manifold: According to [8], [13], Stiefel manifold provides better optimization with quick convergence. Emplying this Stiefel manifold technique, matrice obtained from Stiefel manifold enhances the receiver optimization of the basic and massive MIMO system. Regarding the EE concept, better optimization and quick convergence helps to analyze this research. Also, Stiefel manifold are useful for designing energyefficient feedback.

\section{Massive MIMO and EE Gain}

Many research papers show that MIMO provides EE gain when MIMO system holds more antennas. There are many options which control the energy performance. They depend on how many antennas are in action at the time in either base station or user terminals (mobile station) and quality which is optimization of channel. We can analyze how optimization enhances the EE gain in massive MIMO developments which are wireless networks merged with latest MIMO technology such as manifold and quantization influenced with feedback.
Definition: Spectral efficiency is proportional to the emitted power. So, changes of the EE depend on the power used in MIMO channels.

$$
\mathrm{EE}=\text { Spectral /power }
$$

Regarding the terms used in (3), units of EE, spectral and power of massive MIMO are in (bits/Joule), (bits/channel), (Joule/channel), respectively.

As mentioned in [7], sets of positive semi-definite matrices are considered to identify the Pn-manifold with various trace and ranks, which optimize the overall problems in the massive MIMO system. General Pn-manifolds can be written as:

$$
P_{n}\left(p, \mathbb{F}, \operatorname{Tr}(Q) \leq \rho^{2}, R k(Q)=s\right)
$$

In trace inequalities $\operatorname{Tr}(\mathrm{Q})$, power controls the EE which provides efficiency of the overall massive MIMO system. Finding matrix $\mathrm{Q}$ is appropriate for achieving the goal of this research. This matrix $\mathrm{Q}$ satisfies the capacity requirements on the feedback channel. In MIMO applications, CSI controls the functions of receiver components, which are optimization, equalization and detection. In this research, the feedback link needs better and quick convergence that helps to analyze the EE gain.

\section{FEEDBACK AND CONVERGENCE FOR EE GAIN}

The novelty of feedback designed from manifolds is dependent on the quick convergence. When energy-efficient MIMO and its feedback design with a suitable manifold are considered, fast convergence can be achieved [8]. So, massive MIMO system can be analyzed with feedback and its convergence.

Two types of convergence techniques used in [8] are useful to analyze the EE gain in this research. They are conjugate gradient (CG) and Newton methods which provide convergence. If massive MIMO systems achieve quick convergence, the performance of EE gain will change.

As shown in Fig. 1, massive MIMO has feedback channel that holds the manifold and convergence techniques. MIMO is also considered with the same configuration, but the matrix of the manifold should be larger than expected. Here, rank reduction of the covariance and manifold matrices should be very useful because error performance could be improved [19].

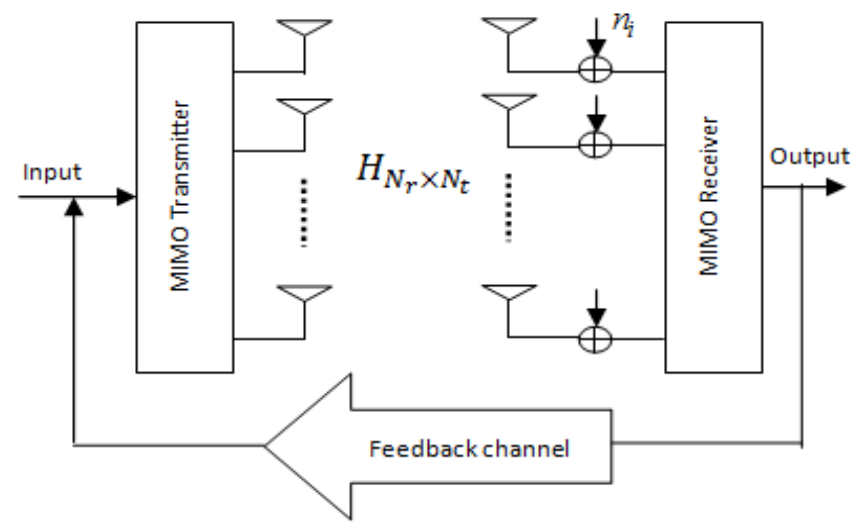

Fig. 1. Massive MIMO Transmitter/Receiver with feedback. 
Most of the applications use the MIMO system with feedback, which needs best EE techniques because many challenges depend on appropriate feedback designs. Here, overall performance of the feedback channel depends on the quantization and minimum MSE, which needs some form of optimization. The capacity requirements of the feedback channel depends on the dynamic channel matrics form the manifolds. An efficient quantization of CSI also provides an enhancement in channel capacity. In order to analyze the EE with massive MIMO applications, studying CSI controls the functions of receiver components, which are optimization, equalization, detection, etc. is important. In order to implement a perfect or limited CSI, the feedback channel should have better optimization technique with quick converging parameters. There are other components such as linear precoding based on CSI, which needs compatible optimization techniques.

In order to optimize the feedback for EE gain, some practical problems are important. They are such as the quantized feedback model and quantization codebook design. Optimization of the quantization influences with these problems, and it uses Stiefel manifold techniques to minimize the problems. Following equation can be used for characterizing the performance when quantized feedback [7] is in an active.

$$
D(w)=E_{H}\left[\lambda_{1}-\max _{\omega \in w} \sum_{i} \lambda_{i}\left|v_{i}^{H} \omega\right|^{2}\right]
$$

Here, $\mathrm{D}(\mathrm{w})$ is the distortion measurement, when feedback channel uses the optimization techniques. In (5), w is the projection codebook and value $\lambda_{1}$ is the largest value of the $H^{H} H$.

\section{A. Feedback Analysis with EE Gain}

Efficiency of receivers and EE gain of massive MIMO depend on the performance of the feedback. In this analysis, there are few assumptions on rank that depends on the channel matrix influenced with noise and environments. Practical limits of antennas used in massive MIMO are directly involved with environmental conditions.

The capacity of the feedback link also increases linearly with the number of transmit antennas [6], [11]. The key problem here is to find algorithms to map frequency domain channel estimates to feedback bits and then from feedback bits to the covariance matrices for all the carriers simultaneously [16].

The MSE and its convergence behavior are considered in the feedback analysis, which transfer the CSI from the receiver to the transmitter. According to [8], convergence of the MSE proved that rank reduction controls the converging time which increases the EE in massive MIMO system.

\section{B. Impact of MSE in EE Gain}

The MSE calculations provide the necessary information to achieve the EE gain through CG algorithm. Convergence behaviour of massive MIMO system depends on the following equation:

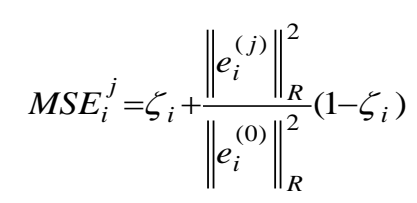

In (6), MSE shows the i-th symbol and j-th iteration when massive MIMO system converges. The $\zeta_{i}$ represents the eigenvalues. According to [18], convergence behavior depends on the covariance matrix $R \in \mathbb{F}^{N_{r} \times N_{r}}$. Convergence rate is the significant concept in this research because it provides necessary information to analyze the EE. It has some influences with $\mathrm{R}$ where properties of eigenvalues take place.

As a conventional technique, CG method is studied. Optimization with minimizing MSE on the Stiefel Manifold is described in [8]. The Stiefel manifold $V_{n, k}^{C}$ is the set of ptuples of orthonormal vectors (7) or equivalently

$$
V_{n, k}^{C}=\left\{Q \in C^{n \times k} \mid Q^{T} Q=I_{k}\right\}
$$

Where $I_{k}$ is the $k \times k$ identity matrix. In Stiefel manifold, $\mathrm{Q}$ is full column rank matrix which has unique solution. According to [13], Stiefel manifold can be employed in the feedback channel of the basic and massive MIMO system where dimensions of matrices could be controlled through this manifold. The space of orthonormal matrices, which is rectangular with $\mathrm{k}<\mathrm{n}$ have associated in definition 1 . The $\mathrm{C}$ used in (7) is representing the complex field of Stiefel manifold.

Definition 1: The definition of complex Stiefel manifold is defined as

$$
\left.Y \in V_{n, k}^{C} \quad\left|\sum_{j}\right| Y_{i, j}\right|^{2}=k / n \quad \forall i
$$

In (8), $\mathrm{k} / \mathrm{n}$ is a constant value on the main diagonal of the matrix, which is a point on the manifold.

Definition 2: The complex dimension of the Stiefel manifold can be defined that it is the sum of the dimension of skew-Hermitian matrices and the dimension of $n \times(n-k)$ matrices.

$$
\operatorname{dim} V_{n, k}^{C}=k(n-k)+0.5 k(k-1)
$$

Signal-to-interference-plus-noise ratio (SINR) and a number of antennas from massive MIMO decide the iterations which take more energy.

$$
\operatorname{SINR} R^{(j+1)}=\frac{P}{\rho^{2}+\frac{P \times N_{t}}{\left(1+\operatorname{SINR} R^{(j)}\right) \times N_{r}}}
$$

In (10), P, is the received power for each transmitting symbol and $\rho^{2}$ the noise power.

$$
M S E^{(j)}=\frac{1}{\left(1+\operatorname{SINR} R^{(j)}\right)}
$$

Equation (11) can be used to calculate the convergence behavior of MSE, which is an error influenced with dimension of the covariance matrix and its rank. 


\section{Impact of Quantization in EE Gain}

As far as this research is concerned, quantization plays the main role in the design of feedback. The convergence and MSE calculations are characterizing the feedback through the correct quantization. The impact of quantization gets changed through the manifolds because it depends on the channel model of massive MIMO.

\section{RESUlTS AND ANALYSIS}

Massive MIMO schemes with Pn-manifold and Stiefel manifolds can provide better EE through the capacity. In order to increase the manifold channel performance, selected configurations of massive MIMO schemes are considered to analyze the results. To reduce the rank and dimension, Stiefel manifold can be employed in the feedback channel of the MIMO scheme. Pn-manifolds can be used in the MIMO channel through the feedback link where quantization technique is applied to characterize channels mathematically.

As shown in Fig. 2, feedback capacity for massive MIMO scheme is increased with antennas, which are in transmitting terminal. Total of antennas from both transmitting and receiving terminals is also important for overall capacity with SNR.

Table I shows the convergence of MSE in massive MIMO schemes. According to [8], Stiefel manifold is applied in both the $\mathrm{CG}$ and Newton methods.

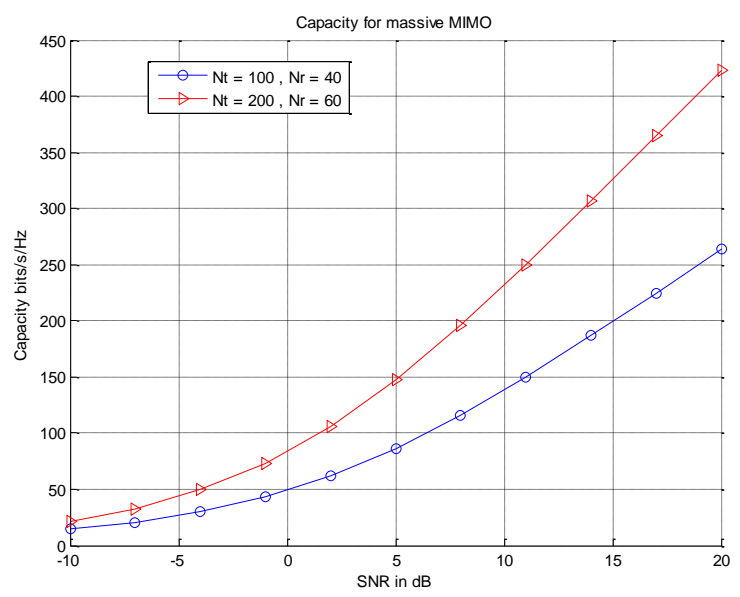

Fig. 2. Feedback capacity for massive MIMO scheme.

TABLE I. CONVERGENCE OF MSE

\begin{tabular}{|l|c|l|}
\hline \multirow{2}{*}{$\begin{array}{l}\text { Massive } \\
\text { MIMO }\end{array}$} & \multicolumn{2}{|l|}{ Stiefel Manifold } \\
\cline { 2 - 3 } & $\boldsymbol{C G}$ & Newton \\
\hline $\begin{array}{c}N_{T}=100 \\
N_{r} \leq N_{T}\end{array}$ & $N_{r} \leq$ Iteration $\leq 2 N_{r}$ & Iteration $\leq 7$ \\
\hline $\begin{array}{c}N_{T}=200 \\
N_{r} \leq N_{T}\end{array}$ & $N_{r} \leq$ Iteration $\leq N_{T}$ & Iteration $\leq 9$ \\
\hline $\begin{array}{c}N_{T}=\infty \\
N_{r} \leq N_{T}\end{array}$ & $2 N_{r} \leq$ Iteration $\leq N_{T}$ & Iteration $\leq 15$ \\
\hline
\end{tabular}

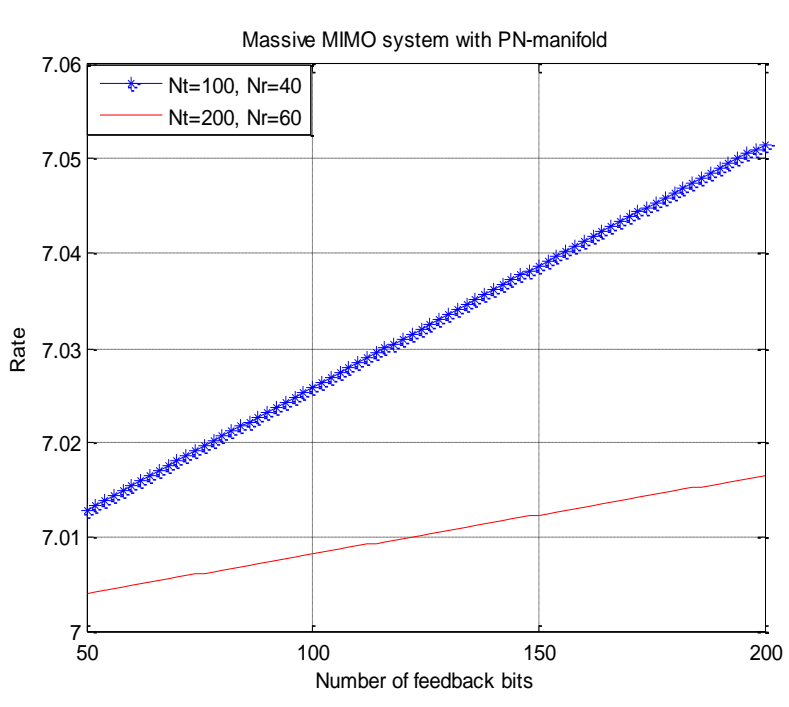

Fig. 3. Rate with feedback bits.

Feedback data can be either sent through the Additive White Gaussian Noise (AWGN) channel or MIMO channel. Quantization is one of the recommended areas in this research. Feedback and quantization in the manifold receiver of MIMO system where each element obtained from Stiefel manifold is quantized with different rate and tested. Computing minimum eigenvectors associated with eigen values is one of the advantages when Stiefel manifold is employed in quantization development. Fig. 3 shows the rate against feedback bits for massive MIMO scheme, which is the new step used to reduce the rank of large matrices employed in the channel matrices.

\section{CONCLUSION AND FURTHER WORK}

In this research paper, we have noted the $\mathrm{EE}$ analysis for massive MIMO wireless network for next generation technology with manifold and convergences techniques used in feedback and quantization. Manifolds are introduced to minimize the dimensions of large matrices created dynamically from the massive MIMO. In particular, Pn-manifold is more attractive to increase the EE gain than the other manifolds because it has more options that are dynamically applicable during the operations. From the theoretical analysis using convergences techniques, quantized feedback can be designed with the Stiefel manifold technique which increases not only the channel capacity, but also the EE gain in the massive MIMO system.

In the future research, we will continue the $\mathrm{EE}$ gain analysis with more results which prove the direct comparison between the EE and manifolds. Also, we can investigate the problem of achievable throughput and EE under complex environments.

\section{ACKNOWLEDGMENT}

The work was funded by the Deanship of Scientific Research (DSR), King Abdulaziz University, Jeddah, under grant No (611-011-D1434). The authors, therefore, acknowledge with thanks DSR technical and financial support. 


\section{REFERENCES}

[1] Hoydis, J, Brink S Ten, and Debbah M, "Massive MIMO in the UL/DL of cellular networks: How many antennas do we need?" IEEE J. Sel. Areas Commun., vol. 31, no. 2, pp. 160-171, 2013.

[2] M. Kobayashi, N. Jindal, and G. Caire, ITraining and feedback optimization for multiuser MIMO downlink," IEEE Trans. Commun., vol.59, no. 8, pp. 2228-2240, Aug. 2011.

[3] Krishnamachari R. T. and Varanasi M. K, "MIMO systems with quantized covariance feedback," IEEE Transactions on Signal Processing, November, 2013.

[4] Krishnamachari R. T. Mahesh K. Varanasi, "MIMO Performance under Covariance Matrix Feedback", IEEE international Symposium on information theory, 2011

[5] Artiga, X., Devillers, B., and Perruisseau-Carrier, J., "Mutual Coupling Effects in Multi-User Massive MIMO Base Stations" Antennas and Propagation Society International Symposium (APSURSI), 2012 IEEE.

[6] Mukkavilli K. K., Sabharwal A, Erkip E, and Aazhang B, "On beamforming with finite rate feedback in multiple-antenna systems," IEEE Trans. Info. Theory, vol. 49, no. 10, pp. 2562-2579, 2003.

[7] Krishnamachari R. T. and Varanasi M. K, "On the geometry and quantization of manifolds of positive semi-definite matrices," IEEE Transactions On Signal Processing, Vol. 61, No. 18, 2013.

[8] Vijey Thayananthan, Ahmed Alzahrani and Iyad Katib, "System Engineering: Optimization on Stiefel Manifold for MIMO System”, Journal of Soft Computing and Software Engineering [JSCSE], 2013

[9] Hoydis, J, Brink S Ten, and Debbah M, "Massive MIMO: How many antennas do we need?" in Proc. of Allerton Conf. on Comm. Control and Comp., 2011, pp. 545-550.

[10] W. Dai, Y. Liu, and B. Rider, "Quantization bounds on Grassmann manifolds and applications to MIMO communications," IEEE Trans.
Inf. Theory, vol. 54, no. 3, pp. 1108-1123, Mar. 2008.

[11] G. Caire, N. Jindal, M. Kobayashi, and N. Ravindran, "Multiuser MIMO achievable rates with downlink training and channel state feedback," IEEE Trans. on Inform. Theory, vol. 56, no. 6, pp. 2845-2866, June 2010.

[12] Krishnamachari R. T. and Varanasi M. K, "Interference Alignment Under Limited Feedback for MIMO Interference Channels," IEEE Transactions On Signal Processing, Vol. 61, No. 15, August 1, 2013.

[13] Henkel O. Sphere packing bounds in the Grassmann and Stiefel manifolds. IEEE Trans. Inf. Theory, 51:3445, 2005.

[14] H'eliot F, Onireti O, and Imran M. A, "An Accurate Closed-Form Approximation of the Energy Efficiency-Spectral Efficiency Trade-Off over the MIMO Rayleigh Fading Channel," in Proc. IEEE ICC'11, 4th International Workshop on Green Comm., Kyoto, Japan, Jun. 2011.

[15] Huh H, Caire G, Papadopoulos H. C, Rampshad S. A, "Achieving large spectral efficiency with TDD and not-so-many base station antennas," in Proc. IEEE Antennas and Propagation in Wireless Communications (APWC), 2011.

[16] Bj"ornson E, Hoydis J, Kountouris M, and Debbah M, "Hardware impairments in large-scale MISO systems: Energy efficiency, estimation, and capacity limits," in Proc. Int. Conf. Digital Signal Process. (DSP), 2013.

[17] Ngo H, Larsson E, and Marzetta T, "Energy and spectral efficiency of very large multiuser MIMO systems," IEEE Trans. Commun., vol. 61, no. 4, pp. 1436-1449, 2013.

[18] J. Tong, P. J. Schreier, and S. R. Weller, "Design and Analysis of Large MIMO Systems With Krylov Subspace Receivers," IEEE Trans. Sig. Proc., vol. 60, No. 5, pp. 2482-2493, 2012.

[19] Y. Sun and M. L. Honig, "Reduced-rank signature-receiver adaptation," IEEE Trans. WirelessComm., vol. 5, no. 10, pp. 2896-2902, Oct. 2006. 